\title{
Personalized genomic medicine: a potential individualized clinical treatment in the future
}

Sheng-Ying Qin

Bio-X Institutes, Shanghai Jiao Tong University, Shanghai 20030, China.

Correspondence to: Prof. Sheng-Ying Qin, Bio-X Institutes, Shanghai Jiao Tong University, 1954 Huashang Road, Shanghai 200030, China. E-mail: chinsir@sjtu.edu.cn

How to cite this article: Qin SY. Personalized genomic medicine: a potential individualized clinical treatment in the future. J Trans/ Genet Genom 2019;3:10. https://doi.org/10.20517/jtgg.2019.05

Received: 19 Aug 2019 Accepted: 19 Aug 2019 Published: 27 Aug 2019

Science Editor: Sheng-Ying Qin Copy Editor: Jia-Jia Meng Production Editor: Tian Zhang

Personalized medicine is a rapidly developing approach in clinical practice that utilizes new technologies to provide decisions in regard to the prediction, prevention, diagnosis and treatment of disease ${ }^{[1]}$. In this special issue of Personalized Genomic Medicine, we highlight certain gene therapy trends and approaches in the field of personalized medicine. The review "MERRF and MELAS: current gene therapy trends and approaches" primarily focuses on the current understanding of the mitochondrial biology principles underlying the mitochondrial DNA (mtDNA) diseases, Myoclonic Epilepsy with Ragged Red Fibers (MERRF) and Mitochondrial Encephalomyopathy, Lactic Acidosis, and Stroke-like Episodes (MELAS). It provides a landscape perspective of gene therapy researches that were devoted to cure these diseases ${ }^{[2]}$. MERRF and MELAS are mitochondrial diseases that are resulted from pathogenic point mutations located within mtDNA region. Currently, there is no cure and patients' care is primarily focusing on treating disease's associated symptoms independently. The specificity of location of mtDNA within the mitochondrial matrix and the inability to correct the disease-causing point mutation added in the difficulty to develop a more effective pharmacological cure. Gene therapy, as an emerging field, seeks to treat or to prevent the disease of interest using viral and/or non-viral modalities by inducing correction of a particular cellular gene via an exogenous payload. The field of gene therapy offer promising solutions that over-perform the traditional symptom management of patients.

In the case report of "Detection of leukocyte adhesion deficiency type 1 in an infant by high-throughput targeted exome sequencing", the authors have highlighted the importance of molecular testing to definitively establish the diagnosis when Leukocyte adhesion deficiency type 1 (LAD-I) was suspected ${ }^{[3]}$. This study thoroughly investigated symptoms and genetic variations of a 43-day-old boy with severe leukocytosis, recurrent infections, defective wound healing and hepatosplenomegaly associated with an acquired

(C) The Author(s) 2019. Open Access This article is licensed under a Creative Commons Attribution 4.0 International License (https://creativecommons.org/licenses/by/4.0/), which permits unrestricted use sharing, adaptation, distribution and reproduction in any medium or format, for any purpose, even commercially, as long as you give appropriate credit to the original author(s) and the source, provide a link to the Creative Commons license, and indicate if changes were made.

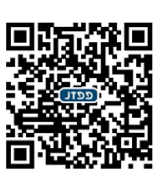


cytomegalovirus infection. To establish the diagnosis definitively, a high-throughput targeted exome sequencing was performed, which was used to make the diagnosis of LAD-I. Moreover, the authors have demonstrated that the early diagnosis of immunodeficiency was essential for optimal management such as hematopoietic stem cell transplantation and rehabilitation outcomes.

In the article "Challenges in implementing genomic medicine: the 100,000 Genomes Project", Barwell et al. ${ }^{[4]}$ evaluated the value of genomic testing in the diagnosis of rare inherited disease in recent years, particularly in presentations when one of a large number of candidate genes may underlie that individual patient's phenotype. The 100,000 Genomes Project aims to change the way patients are diagnosed as well as how personalized treatments and cascading of risk information is carried out. Many important medical conditions may be the result of an inherited mutation in one of a number of different genes. Technical advances have reduced the cost of whole genome sequencing and whole exome sequencing to a level where it is now feasible to analyze multiple genes in one test. Every human carries several hundred potentially pathogenic coding variants, so a major challenge is to understand which of these is relevant to the patient's disease. Data collected from the 100,000 Genomes Project provide useful information to researches on rare diseases and will thus benefit patient care potentially by streamlining the diagnostic process and tailoring care to the individual.

Finally, the special issue of Personalized Genomic Medicine included studies of mental disorder, neurological disease, chronic kidney disease, cancer etc., which explored the genomics testing and gene therapy of individualized clinical treatments. Genomics is progressing from "bench to bedside", which may greatly help us to understand disease mechanisms and identify drug targets once the mutations are found ${ }^{[5,6]}$. Studies of the special issue of Personalized Genomic Medicine reported with combination of genomics and pharmacogenomics testing, gene therapy or other treatment modalities such as stem cell manipulation or xenografts. The field of personalized medicine is experiencing significant expansion. As pointed out by the "Precision Medicine Initiative", it calls for continuous development of personalized medicine which will help patients to receive better treatments using their individual genome characteristics.

\section{DECLARATIONS}

\section{Authors' contributions}

The author contributed solely to the article.

\section{Availability of data and materials}

Not applicable.

\section{Financial support and sponsorship}

None.

\section{Conflicts of interest}

The author declared that there are no conflicts of interest.

\section{Ethical approval and consent to participate}

Not applicable.

\section{Consent for publication}

Not applicable.

\section{Copyright}

(c) The Author(s) 2019. 


\section{REFERENCES}

1. Di Sanzo M, Cipolloni L, Borro M, La Russa R, Santurro A, et al. Clinical Applications of Personalized Medicine: A New Paradigm and Challenge. Curr Pharm Biotechnol 2017;18:194-203.

2. Agresti CA, Halkiadakis PN, Tolias P. MERRF and MELAS: Current Gene Therapy Trends and Approaches. J Transl Genet Genom 2018;2:9.

3. Hong S, Xie LJ, Yang QN, Zhu TW. Detection of Leukocyte Adhesion Deficiency Type 1 In An Infant By High-Throughput Targeted Exome Sequencing. J Transl Genet Genom 2018;2:8.

4. Barwell JG, O’Sullivan RBG, Mansbridge LK, Lowry JM, Dorkins HR. Challenges in Implementing Genomic Medicine: the 100,000 Genomes Project. J Transl Genet Genom 2018;2:13.

5. Olson RE, Cook PS. Genomics: the Clinical Encounter and Parallels Across Complementary and Personalized Medicine. Soc Compass 2018;12:e12621.

6. Zhang L, Hong H. Genomic Discoveries and Personalized Medicine in Neurological Diseases. Pharmaceutics 2015;7:542-53. 